DÉCOLONISATION ET CONSTRUCTION NATIONALE AFRIQUE, ASIE ET QUÉBEC

TITRE: LES NOUVELLES CONSTITUTIONS AFRICAINES: INFLUENCES ET OBJECTIFS. ÉTUDE DE CAS DU BÉNIN, DU GHANA ET DU SÉNÉGAL

Auteur(s): Victor BilodeAu, UniVERsité de SHERBROOKE

Publication: Décolonisation et construction nationale: Afrique, Asie et Québec

PAGE: $48-64$.

Directeurs: PATrick Dramé, PAScal SCAllon-Chouinard et FrançoISE Nozati

ÉditeuR: LES ÉdITIONS DE L'UNIVERSITÉ DE SHERBROOKE, 2016.

ISBN: 978-2-7622-0348-6

URI: HTTP://HDL.HANDLE.NET/11143/8765

DOI: HTTP://DX.DOI.ORG/10.17118/11143/8765 


\section{LES NOUVELLES CONSTITUTIONS AFRICAINES : INFLUENCES ET objectifs. Étude de CAS du BÉNIN, du GHana et du SÉnÉGal}

Victor Bilodeau

La deuxième moitié du XXe siècle est une période de changements importants pour l'Afrique. La grande majorité des colonies accèdent à l'indépendance dans les années 60 et, à la fin du siècle, l'Afrique est complètement décolonisée. Ces nouveaux États, qui ont maintenant le contrôle de leur avenir, passent par une période de questionnement identitaire durant laquelle ils tentent de se définir. La sphère politique n'échappe pas à ce questionnement et plusieurs de ces pays profitent de leur autonomie fraichement acquise pour entreprendre la laborieuse tâche d'élaborer une nouvelle constitution.

Au même moment, le monde des relations internationales est aussi en plein bouleversement. Dans le contexte de la Guerre froide, les États se regroupent principalement en deux grands blocs : celui de l'Ouest, qui s'articule autour des États-Unis, de la France et de la GrandeBretagne; celui de I'Est, qui est mené par I'Union des républiques socialistes soviétiques (URSS) et par la République Populaire de Chine. Certains s'opposent à ce processus de division du monde et mettent sur pied la conférence de Bandung qui se tient en avril 1955. De celle-ci émerge le mouvement des non-alignés qui regroupe les pays refusant de se joindre à l'un des deux grands blocs. Les pères de l'indépendance africaine n'échappent pas à cette division du monde et doivent positionner leurs pays sur la scène internationale.

Considérant ce contexte, peut-il y avoir corrélation entre la position d'un pays et le contenu de sa nouvelle constitution? De plus, si le fait d'être allié officiellement ou du moins idéologiquement à l'un des deux blocs ou encore d'avoir rejoint le mouvement des non-alignés exerce en effet une influence sur le contenu, celle-ci est-elle assez forte pour effacer toute présence de réalités ou d'éléments africains dans ces nouveaux documents constitutionnels? Cette analyse émet l'hypothèse qu'il existe dans ce contexte une corrélation forte entre le positionnement géopolitique des pays africains récemment décolonisés et le contenu de leur nouvelle constitution, mais que cette position n'en conditionne pas directement le contenu. Elle impose plutôt un cadre référentiel et idéologique correspondant à l'alignement au niveau international. Cette position devient une source d'inspiration sur la forme à donner au contenu, mais le fond de ce dernier reste le reflet de réalités et d'objectifs africains et nationaux. 
Afin de vérifier cette hypothèse, trois constitutions seront analysées. Les constitutions ont été sélectionnées à partir de trois critères. Premièrement, la position du pays sur la scène internationale doit être la plus claire possible, ne pouvant que difficilement être remise en doute. Deuxièmement, il convient que toutes les options idéologiques soient représentées, c'est-à-dire qu'il doit y avoir une constitution d'un pays proche du bloc de l'Ouest, une provenant d'un pays proche du bloc de l'Est et une d'un pays proche du mouvement des non-alignés. Troisièmement, les constitutions doivent présenter une certaine stabilité et longévité. Les constitutions choisies seront comparées aux constitutions des États-Unis, de la France, de l'URSS et de la Chine en portant attention aux similitudes ainsi qu'aux points de divergences. Le même processus sera répété, mais cette fois en comparant les différentes constitutions. Dans la mesure du possible, l'étude s'appuie sur des écrits et des discours des présidents en fonction au moment de la rédaction des nouvelles constitutions afin de mieux en saisir les objectifs sous-jacents.

Les trois constitutions retenues pour cette analyse sont celles de la République populaire du Bénin de 1977, celle de la République du Ghana de 1960 et celle de la République du Sénégal de $1963^{2}$. Ces trois textes représentent les trois possibilités de positionnement sur la scène internationale. Au moment de la promulgation de ces constitutions, la République populaire du Bénin était proche du bloc de l'Est, tandis que la République du Sénégal était proche de celui de l'Ouest. Quant à la République du Ghana, elle s'était impliquée dans le mouvement des non-alignés. Ces constitutions respectent aussi le critère de longévité, car celle de la République du Bénin écrite sous la présidence de Mathieu Kérékou est restée en vigueur pendant près de 13 ans, tandis que celle de la République du Ghana, qui a été promulguée sous Kwame Nkrumah, a duré 9 ans. La constitution de la République du Sénégal, écrite alors que Léopold S. Senghor était président, affiche quant à elle la plus grande longévité, car elle est restée en vigueur pendant près de 38 ans.

1. Les constitutions de la France qui sont utilisées sont celles de la IVe République de France de 1946 ainsi que celle de la Ve République de 1958. La constitution de l'U.R.S.S. est celle promulguée en 1936 et la constitution chinoise est celle de la République populaire de Chine de 1954.

2. Les constitutions de I'U.R.S.S., de la Chine et du Bénin proviennent du recueil Constitutions of the countries of the world: a series of updated texts, constitutional chronologies and annotated bibliographies d'Albert P. Blaustein et Gisbert H. Flanz dont il est possible de retrouver un exemplaire mis à jour jusqu'en 2003 à la bibliothèque de la faculté de droit de l'Université de Sherbrooke. La constitution du Ghana provient d'un exemplaire original disponible à la bibliothèque de l'Université Bishop. La constitution du Sénégal provient d'un recueil des textes constitutionnels sénégalais par Ismaïla M. Fall et publié par l'Université Cheikh Anta Diop. La constitution américaine provient du National Constitution Center à Philadelphie. Les constitutions de la France proviennent de la Digithèque MJP de l'Université de Perpignan disponible en ligne. 
L'analyse s'articule autour de trois points. Le premier vise à faire un rapide survol du contexte à l'échelle de chacun des pays afin de voir quelle est la situation politique qui précède la promulgation des nouvelles constitutions et qui caractérise le contexte de leur rédaction. Ensuite, les constitutions seront analysées dans le but de déterminer les caractéristiques des régimes qu'elles mettent en place. Enfin, l'analyse s'attardera sur trois valeurs communes à ces constitutions, soit le socialisme, l'autoritarisme et l'Afrique.

\section{LE CONTEXTE DES NOUVELLES CONSTITUTIONS}

\section{LE BÉNIN : RÉGIONALISME, INSTABILITÉ POLITIQUE ET COUPS MILITAIRES}

Selon Chris Allen, le Bénin dans les années qui précèdent la prise du pouvoir par Kérékou en 1972, est considéré comme l'État africain le moins stable politiquement ${ }^{3}$. II attribue cette situation à deux phénomènes. D'abord, la pratique généralisée du clientélisme qu'il définit ainsi : "Clientelist politics is essentially the exchange of blocks of votes, usually communities or organizations, for valued goods such as a school, piped water or a pay increase 4 . »Cette pratique exerce une pression énorme sur les ressources de l'État comme l'indique l'augmentation du secteur public de $20 \%$ entre 1960-1965 et l'augmentation de $40 \%$ du budget de ce dernier pour la même période ${ }^{5}$. Cette pratique oblige plusieurs gouvernements à adopter de sévères mesures d'austérité. Le deuxième phénomène qui est en partie responsable de l'instabilité politique du pays est le régionalisme. Les résultats des premières élections qui font suite à l'indépendance démontrent bien ce phénomène (voir annexe 1). Le Parti des Nationalistes Dahoméens (P.N.D.) d'Apithy remporte le district du Sud-Est et du Sud, I'Union Démocratique Dahoméenne (U.D.D.) d'Ahomadegbe remporte le Sud-Ouest et le Centre, tandis que le Nord-Est et le Nord-Ouest choisissent le Rassemblement Démocratique Dahoméen (R.D.D.) de Maga. Ce qui est sans doute le plus marquant, c'est la décision du R.D.D. de ne présenter aucun candidat dans les autres districts, puis le fait que seul I'U.D.D. présente un candidat pour s'opposer à Maga, mais dans seulement un des deux districts du Nord où il n'obtient aucun siège. Les militaires renversent à plusieurs reprises les politiciens, ce qui contribue à créer une réelle instabilité gouvernementale. Entre 1960 et 1970, le Bénin va connaître quatre gouvernements civils et quatre coups d'État militaires, dont deux consécutifs en 1967 (voir annexe 2).

3. Chris Allen et al., Benin, the Congo, Burkina Faso, Londres et New York, Pinter Publishers, 1989, p. 16.

4. Ibid., p. 22.

5. Ibid., p. 25. 
Sous le régime du Lieutenant-colonel Alley (1967-1968), des élections sont organisées mais les trois principales personnalités politiques (Apithy - Ahomadegbe - Maga) sont privées du droit de se présenter. Ces dernières invitent la population à boycotter les élections, provoquant un taux de participation de seulement $28 \%$. Alley annule les élections et met Zinsou, un autre politicien, à la tête du pays. II est renversé peu de temps après par un conseil militaire qui organise de nouvelles élections auxquelles Apithy, Ahomadegbe et Maga sont autorisés à participer. Le régionalisme marque encore une fois les résultats des élections (voir annexe 3). Dans le district du Nord-Ouest, les élections sont annulées à cause de violences qui y éclatent. Le conseil militaire prend alors la décision d'installer un triumvirat dans lequel chacun des trois alterne au pouvoir tous les deux ans pendant six ans, alors que les décisions sont prises à deux. Maga accomplit son tour et cède la place à Ahomadegbe, mais pendant que celui-ci est président, Maga et Apithy s'allient pour contrecarrer les décisions d'Ahomadegbe.

En octobre 1972, les jeunes officiers de l'armée renversent le triumvirat, considéré comme un échec, et délogent la vieille garde de l'armée. Mathieu Kérékou, qui fait partie du coup, prend le pouvoir. En 1974, ce dernier déclare l'adoption du marxisme-léninisme et, peu de temps après, il change le nom du pays qui devient la République populaire du Bénin :

[...] as of today, Sunday, 30 November 1975, the militant people of our country solemnly undertake to perpetuate that civilization under new historical conditions and the guidance of the invincible principles of Marxism-Leninism for the honor and dignity of Africa. Therefore, our dear fatherland will be called henceforth the People's Republic of Benin 8 .

Deux ans plus tard, il promulgue une nouvelle constitution qui vise à refléter la nouvelle voie idéologique du pays adopte, soit le marxisme-léninisme.

\section{Le GHANA : L'INDÉPENDANCE et NKRUMAH}

En 1947, I'United Gold Coast Convention (U.G.C.C.), dont fait partie Nkrumah, est fondée. Ce parti occupe la place centrale des négociations avec l'Angleterre pour l'obtention de l'indépendance ${ }^{9}$. Mais, en février et mars 1948, des violences éclatent dans le pays. Certains

\section{Ibid., p. 29.}

7. Jusqu'à ce moment, le Bénin portait encore sont ancien nom colonial, soit le Dahomey.

8. Jeswald W. Salacuse et Albert P. Blaustein, "Adress to the Nation by President Mathieu Kerekou : November 30, 1975 ", dans Albert P. Blaustein et Gisbert H. Flanz, dir., Constitutions of the countries of the worId: a series of updated texts, constitutional chronologies and annotated bibliographies, New York, Dobbs Ferry, vol. 4, 1981, p. 3.

9. A. Mabileau et J. Meyriat, dir., Décolonisation et régimes politiques en Afrique noire, Paris, Armand Colin, 1967, p. 207. 
nationalistes désirent que la marche vers l'indépendance soit plus rapide, ce qui entraîne des dissensions dans le parti U.G.C.C. et pousse Nkrumah à se retirer et à fonder le Convention People's Party (C.P.P.) en $1949^{10}$. Celui-ci va s'imposer dans les années qui suivent au détriment de I'U.G.C.C. Mais le C.P.P. ne fait pas l'unanimité : d'autres partis se forment sur la base d'intérêts régionaux comme le Togoland Congress ou le N.P.P., en 1954, qui représente le Nord du pays et s'oppose au Sud qui détient le pouvoir. En 1954 se tiennent des élections qui portent Nkrumah au pouvoir. Peu de temps après, le National Liberation Movement (N.L.M.) se forme dans la région d'Ashanti, principale productrice de cacao au Ghana, pour contester la politique économique de Nkrumah. Le N.L.M. va demander aux Britanniques de lancer de nouvelles élections et de retarder l'indépendance pour mettre sur pied un projet de constitution fédérative, ce qui aurait assuré une autonomie de la région par rapport aux politiques de Nkrumah qui était alors en négociation avec le pouvoir colonial pour l'obtention d'une constitution unitaire ${ }^{11}$.

En 1956, la Grande-Bretagne décide de trancher le débat sur le terrain. De nouvelles élections sont déclenchées et la voie choisie pour l'indépendance est liée aux résultats. Si le C.P.P. obtient la majorité, le nouveau pays sera unitaire; si le N.L.M l'emporte, le pays sera une fédération. En juillet, le C.P.P. remporte 72 sièges sur 104 avec $57 \%$ des suffrages ${ }^{12}$. En mars 1957, le pays accède à l'indépendance sous une constitution unitaire imposée par les Britanniques.

Dès l'indépendance acquise, Nkrumah s'active à unifier le pays sous sa gouverne personnelle. En 1957, il signe l'Avoidance of Discrimination Act qui interdit les partis à base régionale, tribale et religieuse. Ce décret force les partis de l'opposition à fusionner sous la bannière d'un nouveau parti, à savoir le United Party. Cette fusion neutralise l'opposition. La constitution imposée par les Britanniques contenait plusieurs restrictions quant aux possibilités d'amendements qui visaient à protéger les régions. Mais le Constitution Act de 1958 est venu suspendre ces restrictions, permettant à Nkrumah de supprimer les mesures régionalistes dans les mois suivants, telles que l'abolition des assemblées régionales et le redécoupage

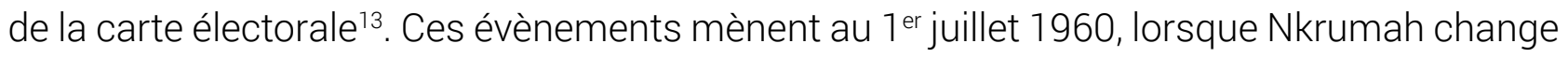
le nom du pays pour la République populaire du Ghana et promulgue une nouvelle constitution $^{14}$.

10. Ibid.

11. Ibid., p. 209.

12. Ibid., p. 209.

13. Ibid., p. 210.

14. Jusqu'à ce moment, le Ghana portait encore sont ancien nom colonial soit la Gold Coast. 
Le Sénégal bénéficie d'une représentation auprès du gouvernement français depuis 1848 . Entre 1872 et 1887, quatre communes de plein droit sont formées, soit celles de Saint-Louis, Gorée, Rufisque et Dakar. La représentation est à l'époque un privilège réservé aux Blancs qui ont un statut de « citoyen » par opposition aux indigènes sénégalais qui ont un statut de « sujet ». II faut attendre 1914 pour voir l'élection du premier représentant noir, à savoir Blaise Diagne. En 1945 est créé un deuxième collège électoral pour les « sujets » qui permet à Senghor de se faire élire. Dans les années qui suivent, Senghor occupe diverses fonctions à titre de député dans le gouvernement français, dont celle de siéger à la commission chargée de rédiger la nouvelle constitution pour la future Ve République de France.

En 1956 est promulguée la loi-cadre qui fait craindre à Senghor la balkanisation de l'Afrique française, d'où l'instigation du projet de former une fédération avec d'autres colonies ${ }^{15}$. En janvier 1959, la constitution fédérale du Mali est adoptée par l'Assemblée constituante fédérale consacrant la naissance de la fédération qui, jusqu'à ce moment, devait avoir quatre participants. Or, peu de temps après, le Dahomey et la Haute-Volta se désistent, par conséquent la Fédération devait désormais être formée seulement du Sénégal et du Soudan. Rapidement se pose le problème de la représentation de la nouvelle fédération au sein de la communauté franco-africaine. Le Sénégal demande que les trois entités soient représentées (le Sénégal, le Soudan, la Fédération), tandis que le Soudan souhaite que seule la Fédération ait ce privilège ${ }^{16}$. De Gaulle affırme quant à lui en privé : " Je connais la République du Soudan, je connais la République du Sénégal, mais la Fédération du Mali connais pas ${ }^{17}$. » Dès août 1960, la Fédération du Mali vole en éclats. Les deux États ne peuvent s'entendre sur le partage du pouvoir exécutif et Modibo Keïta, qui dirige le Soudan, accuse Senghor de privilégier et de protéger les intérêts de la France ${ }^{18}$. À la suite de l'échec de la Fédération, le Sénégal proclame officiellement son indépendance; le Soudan devient lui aussi indépendant sous le nom de République du Mali.

Senghor et son fidèle lieutenant depuis plusieurs années, Mamadou Dia, mettent sur pied une nouvelle constitution dans laquelle Senghor occupe le rôle de président. Mais les pouvoirs exécutifs sont en réalité dans les mains de Dia qui préside le Conseil de gouvernement $^{19}$. Le tandem ne perdure que deux ans. Différents facteurs tels que l'attitude de cer-

15. Adama B. Diop, Le Sénégal à l'heure de l'indépendance. Le projet politique de Mamadou Dia (1957-1962), Paris, L’Harmattan, coll. « Études Africaines », 2007, p. 173.

16. Ibid., p. 181-182.

17. Ibid., p. 180.

18. Ibid., p. 195.

19. Ibid., p. 265. 
tains ministres de Dia, la politique de ce dernier, des conflits au sein de l'Union Progressiste Sénégalaise (U.P.S.), une divergence idéologique entre lui et Senghor et le refus d'octroyer un budget autonome à l'Assemblée expliquent en partie la rupture entre les deux hommes ${ }^{20}$. Le 14 décembre 1962, une motion de censure est déposée à l'Assemblée contre le gouvernement de Dia. Ce dernier demande que la motion soit présentée devant le parti et non devant l'Assemblée, ce que Senghor lui refuse affirmant ainsi la primauté de la Constitution et non du parti. Le 17 décembre, l'Assemblée adopte la motion et le gouvernement de Dia est renversé. Le lendemain, Dia et quatre de ses ministres sont arrêtés pour avoir tenté d'entraver le travail de l'Assemblée en interdisant l'accès au bâtiment et ils sont rapidement condamnés à la prison pour « tentative de coup d'État ${ }^{21}$. » Le 3 mars 1963, Senghor promulgue une nouvelle constitution qui lui octroie les pleins pouvoirs.

\section{LES RÉGIMES ÉTABLIS PAR LES NOUVELLES CONSTITUTIONS}

\section{LE BÉNIN ET LE MARXISME-LÉNINISME}

Le Bénin affirme, dès le préambule de sa constitution, son appartenance à l'idéologie marxiste-léniniste, ce qui suppose une importante influence de la constitution de I'U.R.S.S. sur celle du Bénin. Par contre, l'article 4 vient nuancer cette influence en déclarant : « [...] [Marxisme-léninisme] must be applied in a living, creative manner to the realities of Benin ${ }^{22}$. » Le marxisme-léninisme représente donc une source d'inspiration, mais il est adapté aux réalités locales. Alors que l'article 1 de la constitution de I'U.R.S.S stipule que celle-ci est l'État des travailleurs et des paysans ${ }^{23}$, l'article 2 de la constitution du Bénin affirme plutôt que son État est formé d'une alliance entre toutes les classes de la société dont les paysans et les travailleurs représentent la base ${ }^{24}$. Cette divergence peut en partie expliquer l'originalité de l'Assemblée du Bénin qui répartit ses sièges selon les groupes sociaux. 84 sièges sont réservés aux paysans, 33 aux travailleurs, 8 à la classe moyenne, 20 pour les cadres, 60 pour les fonctionnaires, 25 pour les professeurs, 33 pour l'armée, 21 pour le parti, 46 pour des organisations affiliées au parti et 6 pour les organisations religieuses ${ }^{25}$. La constitution béninoise affirme dans l'article 4 l'adoption du principe du parti-État comme on le retrouve

20. Ibid., p. 275-277.

21. Ibid., p. 279.

22. Jeswald W. Salacuse, et Albert P. Blaustein, «People's Republic of Benin », dans Albert P. Blaustein et Gisbert H. Flanz, dir., loc. cit., p. 3.

23. John N. Hazard, «Union of Soviet Socialist Republics », dans Albert P. Blaustein et Gisbert H. Flanz, dir., op. cit., vol. 20, p. 1.

24. Jeswald W. Salacuse, et Albert P. Blaustein, loc. cit.

25. Chris Allen et al., op. cit., p. 55. 
en U.R.S.S. ${ }^{26}$, ce qui consacre le pouvoir du Parti populaire révolutionnaire sur l'État. Selon l'article 53, l'Assemblée est responsable de l'élection du Président, par contre, les députés de cette même Assemblée sont élus à partir d'une liste qui est déterminée et officialisée par le Comité central du partii ${ }^{27}$. Le président est quant à lui élu à partir d'une liste elle aussi déterminée par le Comité central ${ }^{28}$ (article 53) qui est présidé par Mathieu Kérékou. La présidence qu'institue cette constitution n'a pas beaucoup de pouvoirs. En effet, c'est techniquement l'Assemblée qui est l'organe suprême du gouvernement ${ }^{29}$ et qui possède plusieurs pouvoirs importants; en fait, le pouvoir est entre les mains du parti et de son Comité central, comme le démontre la prérogative qu'il détient sur l'acceptation des candidatures pour les élections. Le Bénin présente donc un régime fortement inspiré de celui de I'U.R.S.S.; les deux États s'articulent autour d'un parti qui dicte en grande partie la politique du pays. Les deux partagent aussi la même base idéologique, mais le Bénin n'hésite pas à adapter l'application de celle-ci à sa propre réalité.

\section{L'ORIGINALITÉ GHANÉENNE}

Le Livre blanc du gouvernement, qui présente le projet de cette nouvelle constitution, affirme que celle-ci «n'est copiée sur aucune Constitution du monde ${ }^{30}$. » Dans son ensemble, celle-ci présente en effet une grande originalité. Par contre, elle semble être bâtie sur un amalgame d'influences extérieures. Le préambule s'ouvre sur la phrase suivante: «We the People of Ghana[.... $]^{31}$ », ce qui se trouve à être une copie exacte de la célèbre phrase d'ouverture de la constitution américaine: "We the People of the United States[...] $]^{32}$. » Mais, elle adopte dès l'article 1A le principe du parti unique représentant le peuple, comme on peut le retrouver, dans une certaine mesure, en U.R.S.S. dans un article qui inscrit le C.P.P comme étant le parti de l'État ${ }^{33}$. La constitution abandonne le principe de la séparation entre le chef de l'État et celui du gouvernement comme on le retrouve en Grande-Bretagne, ce qui avait été imposé par la constitution précédente (article 8$)^{34}$. De plus, cette constitution met sur pied une présidence forte. Le président n'est pas responsable devant l'Assemblée, seulement devant

26. Jeswald W. Salacuse, et Albert P. Blaustein, loc. cit., p. 3.

27. Chris Allen et al., op. cit., p. 55.

28. Jeswald W. Salacuse, et Albert P. Blaustein, loc. cit., p. 10.

29. Ibid., p. 7.

30. A. Mabileau et J. Meyriat, dir., op. cit., p. 211.

31. Ghana, The Constitution of the Republic of Ghana, Accra, The Government Printing Dept, 1960, p. 5.

32. The Constitution of the United States, Philadelphie, National Constitution Center, [s.d.], p. 2.

33. Ghana, op. cit., p. 6.

34. Ibid., p. 7. 
le peuple (article 8.1) ${ }^{35}$. L'article 8.4 stipule que : « [...] the President shall act in his own discretion and shall not be obliged to follow advice tendered by any other person ${ }^{36}$. »

II peut aussi choisir et destituer ses ministres qui sont responsables seulement devant lui et non devant l'Assemblée (article 15 et 16) ${ }^{37}$. II peut dissoudre l'Assemblée s'il le désire, en revanche, cette mesure déclenche automatiquement des élections (article 23.1) ${ }^{38}$. II a aussi un pouvoir de veto sur les lois de l'Assemblée qu'il peut modifier en partie sans pouvoir les rejeter complètement (article 24.1) ${ }^{39}$. Mais le plus intéressant est que tous ces pouvoirs s'accompagnent de la mention suivante: "The power to repeal or alter this article is reserved to the People ${ }^{40}$. » Cette clause implique que le Parlement peut modifier ces dispositions seulement si le peuple lui en a donné l'autorisation par le biais d'un référendum. Or c'est le Président lui-même qui détient le pouvoir de mettre sur pied ces référendums ${ }^{41}$.

Le nouveau régime se caractérise donc par une présidence forte qui bénéficie de plusieurs pouvoirs importants. Mais, en même temps, le monopartisme est imbriqué dans la constitution et le C.P.P. est déclaré le parti de l'État. Ce régime semble être le résultat d'un mélange entre la constitution des États-Unis et celle de I'U.R.S.S.

\section{Le SéNÉGal et la France personnaliséE}

La constitution de 1963 du Sénégal comporte énormément de similarités avec celle de la constitution de la Ve République de France. Les premiers articles de chacune de ces constitutions sont presque identiques. La constitution sénégalaise affirme en effet que : "La République du Sénégal est laïque, démocratique et sociale. Elle assure l'égalité devant la loi de tous les citoyens, sans distinction d'origine, de race, de sexe, de religion. [...] Le principe de la République est : gouvernement du peuple, par le peuple et pour le peuple » (article 1) ${ }^{42}$. Celle de la France stipule quant à elle que : «La France est une République indivisible, laïque, démocratique et sociale. Elle assure l'égalité devant la loi de tous les citoyens sans distinction

35. Ibid.

36. Ibid.

37. Ibid., p. 10-11.

38. Ibid., p. 14.

39. Ibid.

40. Ibid., p. 6-26.

41. Egon Schwelb, "The Republican Constitution of Ghana », The American Journal of Comparative Law, vol. 9 , n० 4 (automne 1960), p. 646-647.

42. Ismaïla M. Fall, dir., Textes constitutionnels du Sénégal, de 1959 à 2007, Dakar, Université Cheikh Anta Diop, coll. « CREDILA», no 23, 2007, p. 58. 
d'origine, de race ou de religion. [...] Son principe est : gouvernement du peuple, par le peuple

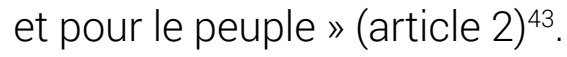

Il est à noter que la phrase « gouvernement du peuple, par le peuple et pour le peuple » est une nouveauté de la constitution de la Ve République de France. II y a donc des emprunts directs à la constitution française qui se retrouvent dans celle du Sénégal; il est important, à ce sujet, de rappeler que Senghor a fait partie de la commission chargée d'écrire la constitution de 1958 de la France. Cependant, en ce qui a trait au président, la constitution sénégalaise prévoit une fonction avec plus de pouvoirs que ceux de son équivalent français. Le président ne partage pas ses pouvoirs avec un premier ministre comme c'est le cas en France et comme c'était le cas avant l'emprisonnement de Dia qui auparavant occupait ce rôle. De plus, le président sénégalais peut instaurer des lois (article 69) ${ }^{44}$, alors qu'en France c'est le premier ministre et non le président qui a ce pouvoir (article 39) ${ }^{45}$. Le conseil des ministres sénégalais est maintenant responsable devant le président et non devant l'Assemblée comme sous l'ancienne constitution (article 43) ${ }^{46}$. La nouvelle constitution n'octroie plus le pouvoir de déposer une motion de censure et l'article 31 affirme que le président doit défendre la constitution ${ }^{47}$.

Donc, bien que la constitution du Sénégal s'inspire fortement de celle de la Ve République de France, il semble que la présidence soit taillée sur mesure pour le « père de la nation » à partir des leçons retenues lors du conflit opposant Dia et Senghor. En effet le poste de président du conseil et le pouvoir de censure sont abolis. D'autre part, les ministres sont responsables devant le président et non devant l'assemblée et ce dernier doit veiller à l'application de la constitution, ainsi que Senghor l'avait fait au moment de son opposition avec Dia qui clamait plutôt la primauté du parti.

\section{LES VALEURS DES NOUVELLES CONSTITUTIONS}

Bien que très différentes, les constitutions béninoise, ghanéenne et sénégalaise présentent des points communs, à savoir une forme de « socialisme à l'africaine », une tendance à l'autoritarisme et un attachement aux spécificités africaines.

43. Université de Perpignan, Digithèque MJP. Digithèque de Matériaux juridiques et politiques, "Constitution du 4 octobre 1958 », [en ligne]http://mjp.univ-perp.fr/france/co1958-0.htm, page consultée le 6 novembre 2011.

44. Ismaïla M. Fall, dir., op. cit., p. 71.

45. Université de Perpignan, loc. cit..

46. Ismaïla M. Fall, dir., op. cit., p. 64.

47. Ibid., p. 63. 
Le socialisme est présent dans les trois constitutions, quel que soit le positionnement du pays au niveau international. Senghor l'inscrit à l'article $1^{48}$ de la constitution sénégalaise et insiste amplement sur son appartenance au "socialisme africain ${ }^{49}$. » De plus, les articles 6 à $20^{50}$ énumèrent une série de droits et de principes en lien avec la famille, le travail, l'éducation, l'égalité, etc. qui ressemblent énormément à ceux garantis par le chapitre 10 de la constitution de I'U.R.S.S. ${ }^{51}$ et sont presque une réplique exacte du chapitre 3 de la constitution chinoise de $1954^{52}$. Le Ghana, de son côté, inscrit aussi le socialisme directement dans sa constitution, soit à l'article 1 A. 1 : « [...] there shall be one National Party which shall be the vanguard of the People in their struggle to build a socialist society [... ${ }^{53}$. » Nkrumah, tout comme Senghor, écrit amplement sur le sujet et ne cache nullement ses orientations idéologiques ${ }^{54}$. Le Bénin affirme ses tendances socialistes encore plus fermement que dans les deux premiers exemples, en déclarant à plusieurs reprises dans sa constitution son appartenance au marxisme-léninisme comme source idéologique.

Ainsi donc, le socialisme est présent dans les trois constitutions, bien que les pays n'adoptent pas nécessairement une même doctrine ou orientation socialiste. Alors que Senghor et Nkrumah prônent le socialisme, Kérékou adopte le Marxisme-Léninisme tout en s'accordant une marge de manœuvre pour diverger de l'idéologie mère en fonction des réalités béninoises. Peut-être peut-on attribuer ces choix de la voie socialiste par l'attrait que représente pour ces nouveaux États le modèle de développement rapide symbolisé par I'U.R.S.S. alors qu'euxmêmes accusent un retard important face au monde occidental?

\section{L'AUTORITARISME}

Une certaine forme d'autoritarisme est présente dans les trois constitutions. Le Ghana et le Bénin inscrivent le monopartisme dans leurs constitutions tandis qu'au Sénégal, bien que légalement il soit permis de former d'autres partis, en réalité, I'U.P.S. n'est pas confronté à une réelle opposition sur le terrain. La constitution béninoise ne met pas sur pied une présidence

48. Ibid., p. 58.

49. Léopold S. Senghor, Liberté 2. Nation et voie africaine du socialisme, Paris, Éditions du Seuil, 1971, p. 252255.

50. Ismaïla M. Fall, dir., op. cit., p. 59-61.

51. John N. Hazard, loc. cit., p. 14-16.

52. Tao-Tai Hsia et Kathryn A. Haun, « People's Republic of China », dans Albert P. Blaustein et Gisbert H. Flanz, dir., op. cit., p. 49-56.

53. Ghana, op. cit., p. 6.

54. Kwame Nkrumah, Africa must unite, London, Mercury Books, 1965, p. 118-131. 
forte, par contre elle pousse plus loin le principe du monopartisme en créant un puissant Parti qui a une forte emprise sur la machine étatique.

L'autoritarisme au Bénin s'explique en grande partie par le régionalisme et l'instabilité politique dont le pays était victime avant la prise du pouvoir par Kérékou. Au Sénégal, Senghor bâtit quant à lui une présidence avec beaucoup de pouvoirs contrairement au modèle de la constitution française. Ses caractéristiques indiquent clairement qu'elle a été mise en place pour éviter que ne se reproduise une situation semblable au conflit avec Dia. Le Ghana est également doté d'une présidence forte, particulièrement sous Nkrumah. L'article 10 inscrit ce dernier dans la constitution comme étant le premier président de la nouvelle république tel que choisi par le peuple aux dernières élections ${ }^{55}$. L'article 55 lui confère le pouvoir d'établir, selon son désir, tout instrument législatif qu'il juge conforme à l'intérêt du pays, ce dernier primant alors sur toute autre législation à l'exception de la constitution ${ }^{56}$. De plus l'alinéa 5 du même article stipule que : «For the purposes of this Article the first President's initial period of office shall be taken to continue until some other person assumes office as President ${ }^{57}$. » Finalement, le dernier alinéa de l'article mentionne que seul le peuple peut révoquer ce pouvoir par un référendum décrété par le Président ${ }^{58}$. Autrement dit, Nkrumah bénéficie de ce pouvoir tant qu'il est à la tête du pays. Ces choix semblent prendre source dans un désir de mettre fin aux revendications de nature régionale ainsi que celles pour un État fédéral.

Bien que ces trois situations autoritaires puissent être liées aux évènements particuliers qui précèdent l'écriture de ces nouvelles constitutions, Nkrumah propose une autre justification de la nécessité pour le Ghana d'être placé sous un régime autoritaire (justification qui peut logiquement être étendue aux trois autres pays), à savoir la nécessité d'assurer le développement : "The reservation of certain powers to the President was felt to be necesssary in order to allow opportunity for decisive action in pushing forward our development ${ }^{59}$. » De plus, il affirme que le Ghana « [...] needed to secure the conditions which would allow us to pursue our policy of reconstruction and development ${ }^{60}$. »

55. Ghana, op. cit., p. 10.

56. Ibid., p. 25.

57. Ibid., p. 26.

58. Ibid., p. 26.

59. Kwame Nkrumah, op. cit., p. 82.

60. Ibid., p.74. 
Dans les trois constitutions, les références directes à l'Afrique et à ses valeurs sont peu nombreuses. Néanmoins, certains éléments valent la peine d'être soulignés. Le préambule de la constitution sénégalaise affirme son désir de voir l'unité africaine se réaliser et affirme que la République ne ménagera aucun effort pour la réalisation de celle-ci ${ }^{61}$. Le Ghana abonde dans le même sens avec l'article 2 qui octroie à l'Assemblée le pouvoir d'abandonner la souveraineté du pays dans le cadre d'un projet ayant pour but l'unification de l'Afrique ${ }^{62}$. II faut aussi souligner la "House of Chiefs ", inscrite dans la constitution ghanéenne sous les articles 49 et 50 , qui est une institution basée sur les chefferies traditionnelles et destinée à gérer ce qui relève de la coutume africaine ${ }^{63}$.

La constitution du Bénin ne fait que très peu référence à l'Afrique à l'exception de la mention de l'adaptation du marxisme-léninisme à la réalité béninoise. Par contre, cette dernière constitution ainsi que la constitution du Sénégal ont l'originalité de garantir le droit à la famille (article 126 pour le Bénin) ${ }^{64}$ (article 14 pour le Sénégal) ${ }^{65}$, qui est une valeur importante dans le cadre de la société africaine précoloniale et qu'on ne retrouve pas dans les autres constitutions qui mettent l'accent sur les droits sociaux et collectifs suivant en cela les constitutions de la Chine et de I'U.R.S.S.

\section{Conclusion}

Il est possible d'affirmer qu'il existe une corrélation forte entre la position du pays sur la scène internationale et le contenu de ces nouvelles constitutions qui se manifeste par l'emprunt d'un cadre référentiel et idéologique. Le cas du Ghana illustre le désir d'originalité de la constitution qui, bien qu'elle semble s'inspirer d'éléments des constitutions du bloc de I'Ouest et du bloc de l'Est, dans son ensemble ne ressemble pas aux constitutions modèles de ces grands blocs. Comme cela a été affirmé dans l'hypothèse originale, les relations avec l'un des deux blocs ou avec le mouvement des non-alignés ne semblent pas être la cause directe du contenu de ces constitutions. Celui-ci serait plutôt la représentation d'objectifs politiques et de réalités africaines et nationales, comme le démontrent l'adaptation des régimes aux situations intérieures et la présence de valeurs communes aux trois constitutions tels le socialisme et l'autoritarisme qui semblent transcender les affiliations idéologiques des pays.

61. Ismaïla M. Fall, dir., op. cit., p. 57.

62. Ghana, op. cit., p. 6.

63. Ibid., p. 24.

64. Jeswald W. Salacuse, et Albert P. Blaustein, loc. cit., p. 21.

65. Ismaïla M. Fall, dir., op. cit., p. 60. 
Les références directes à l'Afrique sont presque complètement absentes des constitutions sauf pour ce qui a trait à la possibilité de voir l'Afrique s'unir ou la survivance des chefs traditionnels dans la politique ghanéenne. Par contre, les constitutions du Sénégal et du Bénin font indirectement référence à l'Afrique traditionnelle en affirmant l'importance de protéger la famille dans la constitution. II serait aussi possible d'affirmer que la présence même du socialisme dans ces constitutions pourrait être indirectement rattachée aux pratiques de la communauté traditionnelle africaine. Finalement, les constitutions mettent sur pied des présidences relativement fortes où le président cumule les fonctions de chef de l'État et de chef de l'exécutif, ce qui pourrait être perçu comme la survivance et l'héritage de la culture du chef telle qu'on pouvait la concevoir en Afrique traditionnelle. 


\section{AnNeXe 1}

RÉSULTATS DES ÉLECTIONS DE $1960^{66}$

\begin{tabular}{|c|c|c|c|c|c|c|c|}
\hline \multirow{2}{*}{ District } & \multirow{2}{*}{$\begin{array}{c}\text { Total } \\
\text { sièges }\end{array}$} & \multicolumn{2}{|c|}{ PND } & \multicolumn{2}{c|}{ UDD } & \multicolumn{2}{c|}{ RDD } \\
\cline { 3 - 8 } & Votes & Sièges & Votes & Sièges & Votes & Sièges \\
\hline Sud-Est & 45 & 53912 & 45 & 13432 & 0 & 0 & 0 \\
\hline Sud & 35 & 30121 & 20 & 38690 & 15 & 0 & 0 \\
\hline Sud-Ouest & 30 & 24884 & 9 & 33120 & 21 & 0 & 0 \\
\hline Centre & 45 & 24971 & 10 & 66931 & 35 & 0 & 0 \\
\hline Nord-Est & 30 & 0 & 0 & 0 & 0 & 43184 & 30 \\
\hline Nord-Ouest & 40 & 0 & 0 & 6214 & 0 & 49970 & 40 \\
\hline Total & 225 & 133888 & 84 & 158477 & 71 & 87154 & 70 \\
\hline
\end{tabular}

Note : Les « 0 » indiquent que le parti n’a pas présenté de candidat dans ce district.

\section{AnNexe 2}

Liste des gouvernements du Dahomey 1960-1970

\begin{tabular}{|c|c|c|}
\hline 1. & $1960-1963$ & Hubert Maga \\
\hline 2. & $1963-1964$ & Coup militaire - Colonel Christophe Soglo \\
\hline 3. & $1964-1965$ & Sourou Migan Apithy \\
\hline 4. & 1965 & Tahirou Congako \\
\hline 5. & $1965-1967$ & Coup militaire - Général Christophe Soglo \\
\hline 6. & $1967-1968$ & Coup militaire - Lieutenant-colonel Alphonse Alley \\
\hline 7. & $1968-1969$ & Émile Derlin Zinsou \\
\hline 8. & $1969-1970$ & Coup militaire - Conseil militaire \\
\hline
\end{tabular}

66. Samuel Decalo, «Regionalism, Politics, and the Military in Dahomey », The Journal of Developing Areas, vol. 7, n³ (avril 1973), p. 454. 
Annexe 3

RÉSULTATS DES ÉLECTIONS DE $1970^{67}$

\begin{tabular}{|c|c|c|c|c|}
\hline District & Ahomadegbe & Apithy & Maga & Zinsou \\
\hline Sud-Est & $8 \%$ & $82 \%$ & $5 \%$ & $3 \%$ \\
\hline Sud & $51 \%$ & $35 \%$ & $6 \%$ & $5 \%$ \\
\hline Sud-Ouest & $43 \%$ & $27 \%$ & $8 \%$ & $6 \%$ \\
\hline Centre & $73 \%$ & $19 \%$ & $5 \%$ & $3 \%$ \\
\hline Nord-Est & $2 \%$ & - & $97 \%$ & - \\
\hline Nord-Ouest & \multicolumn{4}{|c|}{ Élections annulées } \\
\hline
\end{tabular}

67. Chris Allen et al., op. cit., p. 30. 


\section{SOURCES ET BIBLIOGRAPHIE}

[s. a.]. The Constitution of the United States. Philadelphie, National Constitution Center, [s.d.], 19 p. [en ligne]http://constitutioncenter.org/media/files/constitution-full-text.pdf.

[s. a.]. The Constitution of the Republic of Ghana. Accra, The Government Printing Dept, 1960, 25 p.

ALLEN, Chris, RADU, Michael S., SOMERVILLE, Keith et Joan BAXTER. Benin, the Congo, Burkina Faso, Londres/New York, Pinter Publishers, coll. « Marxist regimes series », 1989, 300 p.

BLAUSTEIN, Albert P. et Gisbert H. FLANZ. Constitutions of the countries of the world. A series of updated texts, constitutional chronologies and annotated bibliographies. Dobbs Ferry, Oceana Publications, 2003.

DECALO, Samuel. « Regionalism, Politics, and the Military in Dahomey ». The Journal of Developing Areas, vol. 7, n³ (avril 1973), p. 449-487.

DIOP, Adama B. Le Sénégal à l'heure de l'indépendance : Le projet politique de Mamadou Dia (19571962). Paris, L'Harmattan, 2007, 289 p.

FALL, Ismaïla M., dir. Textes constitutionnels du Sénégal, de 1959 à 2007. Dakar, Université Cheikh Anta Diop, coll. «CREDILA», n²3, 2007, 257 p.

MABILEAU, A. et J. MEYRIAT, dir. Décolonisation et régimes politiques en Afrique noire. Paris, Armand Colin, 1967, $276 \mathrm{p}$.

SCHWELB, Egon. "The Republican Constitution of Ghana ». The American Journal of Comparative Law, vol. 9, nº 4 (automne 1960), p. 634-656.

SENGHOR, Léopold S. Liberté 2. Nation et voie africaine du socialisme. Paris, Éditions du Seuil, 1971, $315 \mathrm{p}$.

Université de Perpignan, Digithèque MJP. Digithèque de Matériaux juridiques et politiques. «Constitution du 4 octobre 1958 ». [en ligne]http://mjp.univ-perp.fr/france/co1958-0.htm, page consultée le 6 novembre 2011. 